- Lon Schneider, an Alzheimer's researcher at the University of Southern California in Los Angeles, questions the decision to start large trials before the drugs, and the amyloid hypothesis, have been well validated. "Why are there so many antibodies when none so far have proven efficacy?" he asks, noting that behavioural interventions, such as diet and exercise, have been shown to slow Alzheimer's as much as any drug (A. M. Clarfeld and T. Dwolatzky JAMA Intern. Med. 173, 901-902; 2013).

But not everyone agrees. "This is the time to be bold," says Randall Bateman, a neurologist at Washington University in St. Louis, Missouri. "It seems to me the cost of delay from a human-suffering standpoint is much more expensive than the cost of moving forward."

Bateman is leading a trial that is testing solanezumab and ganetenerumab - developed by Roche of Basel, Switzerland - in 160 people between 18 and 80 years old who have a genetic risk of Alzheimer's, but no symptoms. It is one of several efforts attempting to determine whether the disease can be prevented by destroying amyloid protein before the brain is damaged. That harm occurs over decades (R. J. Bateman et al. N. Engl. J. Med. 367, 895-804; 2012), and many Alzheimer's researchers suspect that antibody-drug trials have failed because they have treated people too late.

This hypothesis is supported by Lilly's finding that only people with mild disease benefit from solanezumab. The latest results also demonstrate for the first time in humans that slowing amyloid deposition can slow down cognitive decline, says Eric Reiman, executive director of the Banner Alzheimer's Institute in Phoenix, Arizona.

That is important because the US Food and Drug Administration has said that it will not approve drugs that block amyloid deposits without sufficient evidence of a clinical benefit. If one drug company can prove the cause and effect between amyloid accumulation and Alzheimer's progression, all companies will benefit, says Reiman, who is leading a trial of crenezumab, a Roche drug that has also failed previously in large trials.

If such drugs falter in larger preventative trials, that would be a setback for Alzheimer's research in general, says Gandy. "The main concern is that the pipeline behind amyloid-reducing agents is really pretty spare," he says. However, at least three companies are developing treatments - some of which are antibody drugs - that target a different protein, tau, which destroys neurons in advanced Alzheimer's disease. See go.nature.com/lathpu for a longer version of this story.

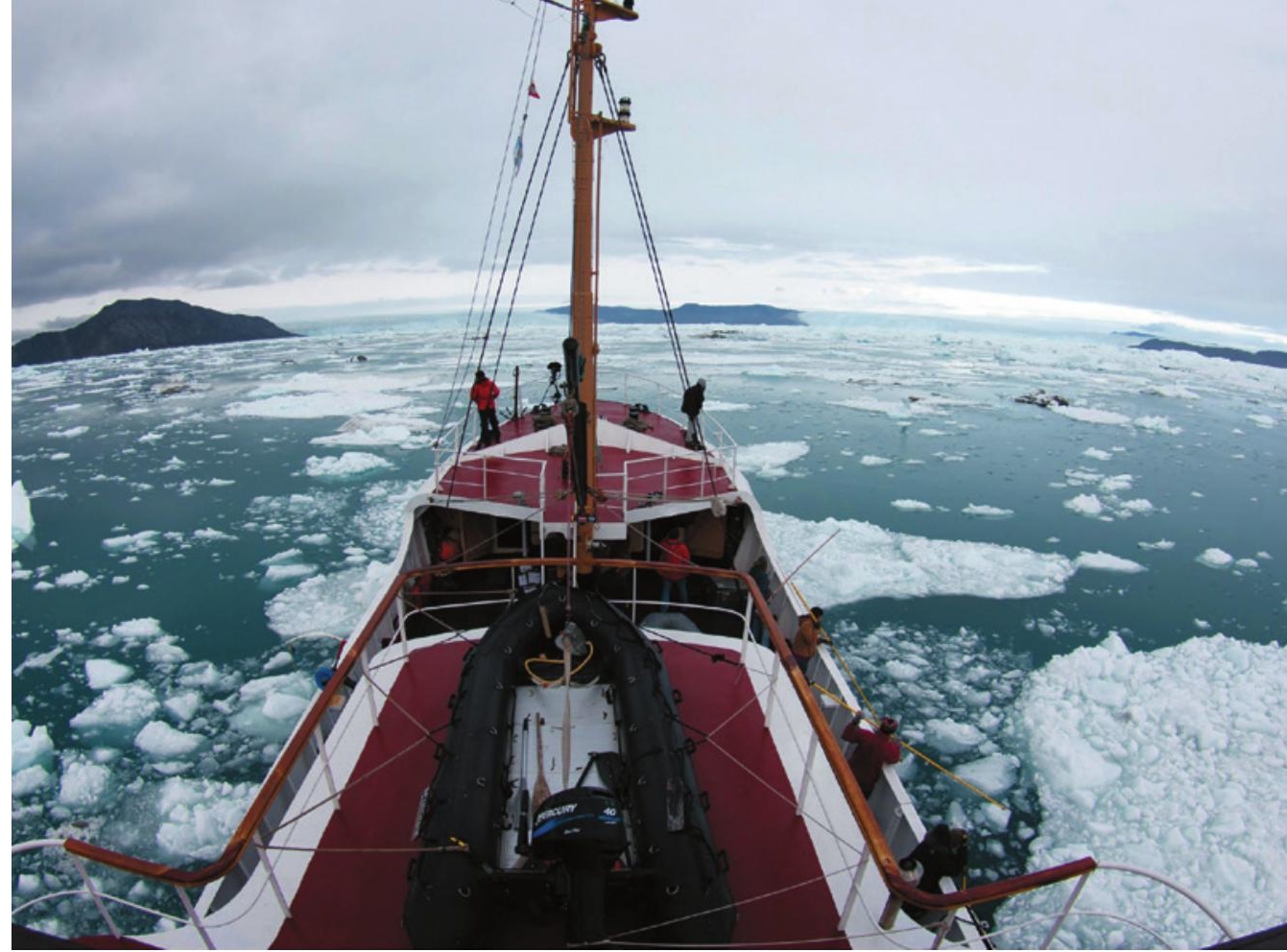

The MV Cape Race is using sonar to map the depth of water around Greenland's west coast.

GLACIOLOGY

\title{
NASA launches mission to Greenland
}

\author{
Ship and planes will probe water-ice interface in fjords.
}

\section{BY JEFF TOLLEFSON}

$\mathrm{W}$

hen the retired fishing trawler MV Cape Race sets off along Greenland's west coast this week, it will start hauling in a scientific catch that promises to improve projections of how the ice-covered island will fare in a warming world. The ship's cruise is the initial phase of a six-year air and sea campaign to probe interactions between Greenland's glaciers and the deep, narrow fjords where they come to an end.

Called Oceans Melting Greenland (OMG), the US\$30-million NASA project will help scientists to predict the future of the Greenland ice sheet, which holds enough water to boost sea levels by around 6 metres and already seems to be melting more rapidly in response to increasing air temperatures. But it is not clear how much the oceans affect the rate of melting along the island's edges, which depends on poorly known variables such as how warm, saline water interacts with the glaciers.

"It should be a powerful constraint on our knowledge and ability to model ice loss there," says principal investigator Joshua Willis, an oceanographer at NASA's Jet Propulsion Laboratory in Pasadena, California.

When simulating glacier dynamics, current global climate models consider only ice's interactions with the atmosphere, says William Lipscomb, an ice modeller at Los Alamos National Laboratory in New Mexico. He is working to incorporate ice-ocean interactions around Antarctica into a climate model being developed by the US Department of Energy. But in Greenland, the intricately carved coastline makes this much more difficult. The department plans to give researchers at the Naval Postgraduate School in Monterey, California, $\$ 466,000$ over 2 years to build a detailed model that will link the land ice and oceans around Greenland. OMG data will help to validate that model, says project leader Frank Giraldo.

Work by OMG participant Eric Rignot, a glaciologist at the University of California, Irvine, underscores the importance of detailed data (E. Rignot et al. Geophys. Res. Lett. http:// doi.org/6dn; 2015). Using sonar data from one part of western Greenland, Rignot's team found that existing maps underestimate the depth of three fjords by several hundred metres. It also found that glaciers flowing into all three fjords extended deeper than was thought, far enough below fresh surface waters to reach a warm, salty layer flowing up from the Atlantic Ocean that could accelerate melting and contribute more to sea-level rise than had been believed.

"With OMG, we are going to reveal the depth of these fjords," says Rignot. 
The programme will also provide valuable information on the physical characteristics of glacier ice. Last December, geophysicist Beata Csatho of the University at Buffalo in New York and her colleagues reported using surfaceelevation data to estimate how much ice mass Greenland had lost between 1993 and 2012 (B. M. Csatho et al. Proc. Natl Acad. Sci. USA 111, 18478-18483; 2014). The data were fairly reliable over the island's interior, Csatho says, but measurements were more difficult along its edges, where the ice tends to be warmer, thicker and full of crevices. "It's still a challenge to get the mass of these glaciers," she says.

When the aerial phase of OMG begins next year, planes will fly inland from the coast, taking measurements of slight changes in gravitational pull that can be used to produce low-resolution maps of the topography under both water and ice. Planes will also drop more than 200 temperature and salinity probes into fjords and coastal waters, and take radar measurements along the coast to track large-scale ice loss over five years. Analysing that ice loss in light of the new topographical and oceanographic data will help researchers to determine where, and to what extent, deeper saltwater currents affect glaciers.

Lipscomb says that all these OMG data should help modellers as they incorporate ocean-ice interactions around Greenland into their models. That work is still in its early stages, he says, "but the data that they are getting in this project is exactly what we need".

\section{Kepler spies most Earth-like planet yet}

\section{NASA mission finds a potentially rocky world orbiting a star that resembles the Sun.}

\section{BY ALEXANDRA WITZE}

$\mathrm{E}$ ven as astronomers are reporting what looks like the closest thing yet to an Earth-like exoplanet, NASA is winding down the prolific Kepler mission that made the find. Sometime next year, team scientists plan to release their final list of planet discoveries.

On 23 July, the Kepler team announced the existence of a planet 1.6 times the size of Earth, orbiting a Sun-like star 430 parsecs away (J. M. Jenkins et al. Astron. J. 150, 56, 2015). The planet, named Kepler-452b, is in the habitable zone, orbiting its star at a distance where liquid water could exist. Team scientists say that there is a little more than a $50 \%$ chance that the planet is rocky, which would make it the closest thing to a true Earth analogue yet discovered.

Kepler's latest batch of discoveries also includes at least 11 other planets that are all less than twice the size of Earth and orbit in their stars' habitable zones. But Kepler-452b's star is slightly brighter than the Sun, in contrast to the cool, dim stars that host other known Earth-sized planets.

"It is the first terrestrial planet in the habitable zone around a star very similar to the Sun," says Douglas Caldwell, an astronomer at the SETI Institute in Mountain View, California.

Scientists cannot measure the mass of Kepler-452b directly, but modelling suggests that the planet is probably five times the mass

\section{HABITABLE HUNT}

NASA's Kepler mission has found several small planets outside the Solar System that could harbour

life. They orbit their stars in the 'habitable zone' where liquid water could exist.

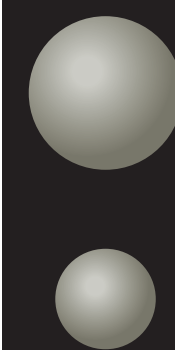

Habitable zone

Kepler-452b

Announced:

Announced: July 2015

Diameter: $\quad 60 \%$ larger

Orbits its star: Once every

385 Earth days

Kepler-186f

Announced: April 2014

Diameter: $\quad 10 \%$ larger

Diameter: $10 \%$ larger

Orbits its star: Once every

130 Earth days

Earth

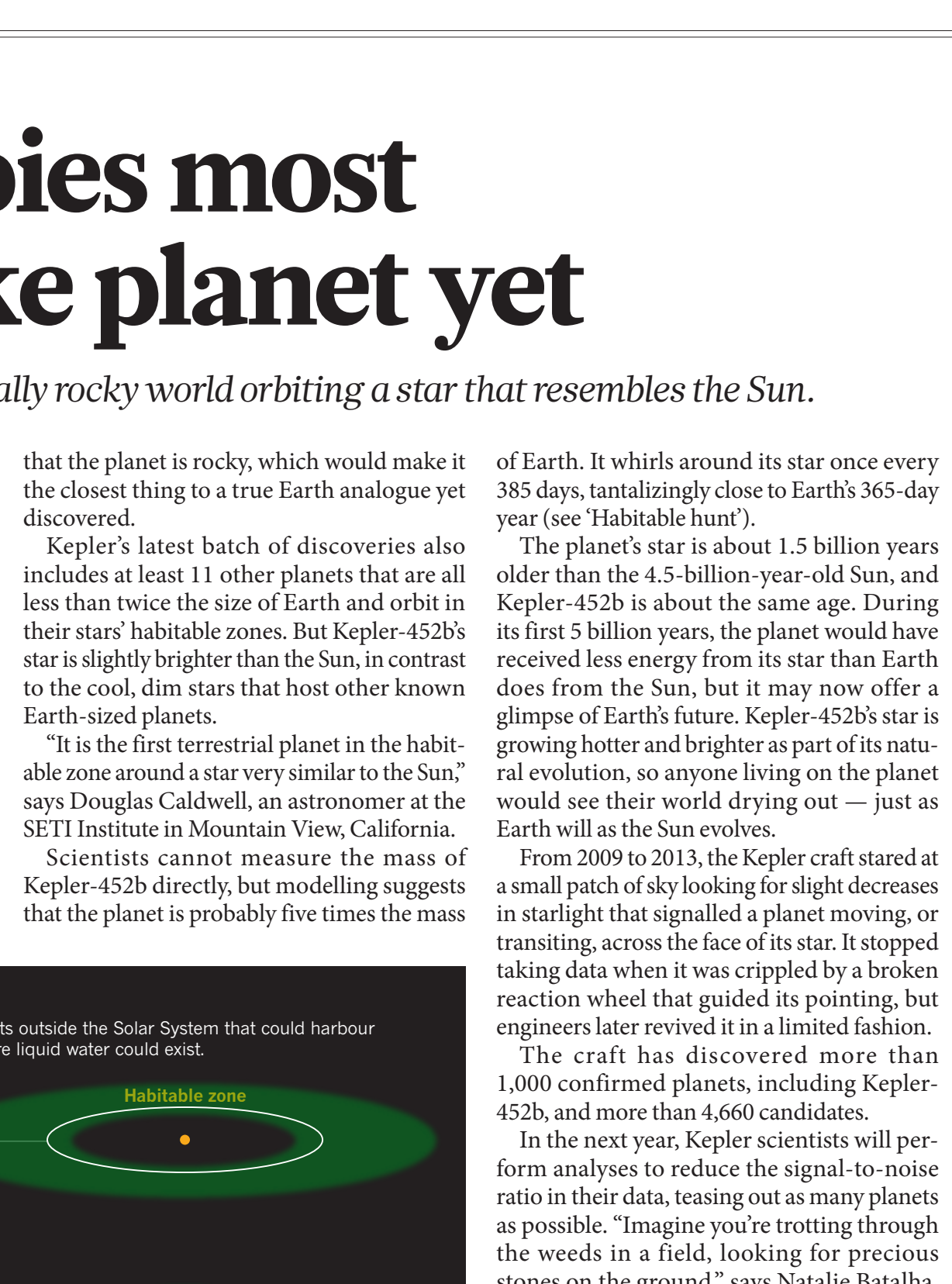

